\title{
THE COGNITIVE ORIENTATION OF INTUITIVE THINKING
}

\author{
Shulamith Kreitler, \& Carmit Benbenisty \\ School of Psychological Sciences, Tel-Aviv University (Israel)
}

\begin{abstract}
The objective of the study was to identify the motivational components of intuition. The methodology was based on the cognitive orientation (CO) theory which assumes that cognitions orient toward behaviors. The hypotheses were that that the $\mathrm{CO}$ assessment of intuition will be related to the standard measures of intuition. The participants were 90 undergraduates in the behavioral sciences. They were administered the $\mathrm{CO}$ questionnaire of intuition that included beliefs of four types. The Types of Intuition scale and the Decision Style scale. The results showed that the four CO types of beliefs predicted significantly all the intuition measures. The major motivational components of intuition were found to be focusing on emotions, opening-up to situations, preference for fast and easy solutions and self reliance. The theoretical conclusions are that intuition is a personality tendency grounded in a specific motivational network, amenable for assessment and training.
\end{abstract}

Keywords: Intuition, cognitive orientation, beliefs, motivation.

\section{Introduction}

Intuitive thinking is a common type of thinking, usually considered as grounded in unconscious, illogical, uncontrolled, emotionally-tainted cognition, involved in various cognitive activities, including decision making, planning, problem solving, mathematics and religion (Heintzeloman \& King, 2016; Sagiv et al., 2014). On the one hand, it has been claimed to be responsible for biases (4), overconfidence in judgments (Hansson et al., 2008), wrong conclusions in diagnoses (Gäbler, 2017), but on the other hand, it was reported as correlated with creativity, humor, aesthetic judgment, empathy and social popularity (Norris \& Epstein, 2018). It is described as fast, effortless, and easy as compared with rational, evidence-based and deliberate thinking (Kahneman, 2003). It appears already in children (Schlottmann, 2001) and in animals (Rakoczy et al., 2014) and is manifested neurally in desynchronized alpha waves in the posterior cortex (Razumnikova et al., 2017). Sometimes it is considered as secondary to rational thinking which it complements (Thompson, Prowse Turner, \& Pennycook, 2011), at other times as a separate experientially-based kind of cognition, opposed to logical deliberation (Kahneman, 2003). Intuition is subject to individual differences but little is known about its psychological correlates. It is the objective of this study to examine the motivational correlates of intuition which would provide insight into the tendencies underlying intuition, enabling identifying individuals who rely on intuition and even training those who do not.

\section{The theoretical approach}

\subsection{The cognitive orientation theory}

The cognitive orientation (CO) approach is a theory and methodology which provides an explanation about why individuals behave as they do without relying on assumptions of consciously controlled systematic decision making based on considerations of benefit and utility (Kreitler, 2004; Kreitler \& Kreitler, 1982). Rather, it is assumed that behavior is function of specific beliefs that orient toward the behavior without the conscious intention or decision of the individual. The beliefs are characterized in terms of form and contents. Formally the beliefs are of four kinds: beliefs about oneself that describe traits, events and other facts referring to oneself; general beliefs that deal with information about others and reality in general; beliefs about rules and norms which refer to ethical, social, esthetical and functional rules and standards; and goal beliefs which refer to actions or states desired or undesired by the individual. In terms of contents the beliefs refer to deep underlying meanings of the involved inputs rather than to their obvious and explicit surface meanings. These underlying meanings are identified for the 
behavior in question by means of a standard stepwise interviewing procedure conducted with pretest subjects. The recurrent meanings in these interviews are called motives and are used for formulating statements conforming to the four types of beliefs. For example, a motive such as overlooking details could be formulated in the following statements: "I dislike dealing with details", "Details are unimportant", One should never overlook the details" and "I would like to be able to deal with details", referring to beliefs about self, general beliefs, norm beliefs and goal beliefs, respectively. The meaning elaborations are based on clarifying the extent to which the involved beliefs support or do not support the indicated action. If all four or at least three belief types support the action, a cluster of beliefs is formed orienting toward the particular act. It generates a unified tendency or vector which represents the motivational disposition orienting toward the performance of the action. The beliefs are assessed in terms of a $\mathrm{CO}$ questionnaire which provides scores for the four belief types (see Tools and Appendix). Previous studies showed that questionnaires constructed on the basis of the $\mathrm{CO}$ theory provided significant predictions of various cognitive, emotional, behavioral and physiologic variables (Kreitler, 2004, 2014; Kreitler \& Kreitler, 1982).

\subsection{The hypotheses}

The hypotheses of the study were that the scores of the CO questionnaire of intuition -the four belief types and the factors based on the motives - will be correlated with the scores of intuition on the basis of responses to validated questionnaires assessing intuition. The expectation is that all four belief types or at least three will have significant contributions (Kreitler, 2004).

\section{Method}

\subsection{Design}

The study was based on a correlational design. It consisted in analyzing the correlations between the scores of the $\mathrm{CO}$ questionnaire of intuition, on the one hand, and the scores of intuition provided by two intuition scales, on the other hand.

\subsection{Participants}

The participants were 90 undergraduate students ( 55 women, $45 \mathrm{men}$ ) in the behavioral sciences, in the age range 21-25 years. They were administered three questionnaires in random order, in the google.docs online format. Questionnaires that had over $5 \%$ of missing responses $(16 \%)$ were deleted from the final file.

\subsection{Tools}

There were two questionnaires assessing intuition. Both underwent extensive validation examinations. One was the The Types of Intuition Scale (TIntS) (Pretz, Brookings, Carlson et al., 2014). The scale includes 29 items in the form of statements to which the respondents were asked to respond by checking one of 5 alternatives ranging from definitely false $(=1)$ to definitely true $(=5)$. The scale measures three types of intuition identified on the basis of a previous literature review): holistic ( 8 items), inferential (12 items), and affective ( 9 items). Holistic intuitions integrate diverse sources of information in a Gestalt-like, non-analytical manner; inferential intuitions are based on previously analytical processes that have become automatic; and affective intuitions are based on feelings.

The second intuition scale was the Decision Style Scale (DSS) (Hamilton, Shih \& Mohammed, 2016) DSS representing rational and intuitive dimensions. The rational decision style involves information gathering, alternative generation, and thorough evaluation; the intuitive style involves relying on feelings and making decisions quickly. Each of the two dimensions was represented by five items. The response format was a 5-point Likert scale ranging from 1 (strongly disagree) to 5 (strongly agree).

A third questionnaire the subjects got was the CO questionnaire of intuition (see Appendix). It consisted of four parts corresponding to eh four belief types with 18 items in each. The response format consisted of 4 response alternatives ranging from not at all tru $=1)$ to very true $(=4)$. Thus, each individual got four scores for beliefs bout self, general beliefs, no32.7\% (affective i8ntuition). rms and goals. The 18 items in each part of the questionnaire represented 18 motives. These yielded five factors (principal component; accounting for $68 \%$ of the variance) labelled as: emotions, opening-up, fast solutions, comprehensive view, self reliance. Thus, each subject got also five scores for the five motive factors. 


\section{Results}

\subsection{Control analyses}

Control analyses showed that all the five dependent measures of intuition and the four basic intuition measures of the $\mathrm{CO}$ questionnaire had satisfactory reliability coefficients $(\alpha$ Cronbach $\geq .70$ ). Further, correlation analyses showed that of the four belief types of the $\mathrm{CO}$ questionnaire only beliefs about self and goal beliefs were correlated significantly $(\mathrm{r}=.25, \mathrm{p}<.05)$. Concerning the dependent variables assessing intuition, there were the following significant correlations: holistic intuition (TIntS) with intuition (DSS) $\mathrm{r}=.35(\mathrm{p}<.01)$, affective (TIntS) with intuition (DSS) $\mathrm{r}=41 \quad(\mathrm{p}<.01)$, and holistic (TIntS) with rationality (DSS) $\mathrm{r}=-26(\mathrm{p}<.05)$.

\subsection{Relations between the $\mathrm{CO}$ of Intuition and the intuition scales}

Table 1 presents the results of the regression analyses performed with the four types of CO beliefs as predictors and each of the five variables assessed by the two questionnaires TIntS and DSS as dependent variables. The results show that the CO beliefs yielded a significant prediction of all the four intuition variables. The amount of variance in the intuition variables accounted for by the $\mathrm{CO}$ beliefs ranged from $26.2 \%$ (holistic intuition) to $32.7 \%$ (affective intuition). On the whole the differences in the variance accounted for are not large (on the average $28.67 \%$ ). Thus, the results support the hypothesis in showing that the $\mathrm{CO}$ beliefs are related significantly to the intuition variables.

Table 1. The results of regression analyses between the CO four belief scores and the variables assessing intuition (TIntS and DSS).

\begin{tabular}{|c|c|c|c|c|}
\hline & \multicolumn{4}{|c|}{$\begin{array}{lll}\text { Dependent variable: Holistic (TIntS) } & \mathrm{R}=.544 & \mathrm{R}^{2}=.296 \\
\mathrm{~F}(\mathrm{df}=4 / 85)=4.21^{* *} & & \end{array}$} \\
\hline Coefficients & unstandardized & Standardized & t-test & \\
\hline Beliefs about self & .367 & .345 & & $2.370 * * *$ \\
\hline General beliefs & .252 & .221 & & $2.000^{*}$ \\
\hline Beliefs about norms & .342 & .319 & & $2.381 * *$ \\
\hline \multirow[t]{2}{*}{ Goal beliefs } & .332 & .238 & & $2.202 *$ \\
\hline & \multicolumn{4}{|c|}{$\begin{array}{lll}\text { Dependent variable: Inferential (TIntS) } & \mathrm{R}=.512 & \mathrm{R}^{2}=.262 \\
\mathrm{~F}(\mathrm{df}=4 / 85)=3.07^{*}\end{array}$} \\
\hline Beliefs about self & .200 & .198 & & $2.036 *$ \\
\hline General beliefs & .252 & .221 & & $2.000^{*}$ \\
\hline Beliefs about norms & .304 & .286 & & $2.270^{*}$ \\
\hline \multirow[t]{2}{*}{ Goal beliefs } & .299 & .257 & & $2.183^{*}$ \\
\hline & \multicolumn{4}{|c|}{$\begin{array}{l}\text { Dependent variable: Affective (TIntS) } \mathrm{R}=.572 \mathrm{R}^{2}=.327 \\
\mathrm{~F}(\mathrm{df}=4 / 85)=3.66^{* *}\end{array}$} \\
\hline Beliefs about self & .317 & .335 & & $2.530 * *$ \\
\hline General beliefs & .372 & .322 & & $2.025^{*}$ \\
\hline Beliefs about norms & .358 & .320 & & $2.350^{*}$ \\
\hline \multirow[t]{2}{*}{ Goal beliefs } & .301 & .275 & & $2.110^{*}$ \\
\hline & \multicolumn{4}{|c|}{$\begin{array}{l}\text { Dependent variable: Intuitive (DSS) } \mathrm{R}=.512 \mathrm{R}^{2}=.262 \\
\mathrm{~F}(\mathrm{df}=4 / 85)=3.68^{* *}\end{array}$} \\
\hline Beliefs about self & .349 & .295 & & $2.451^{* *}$ \\
\hline General beliefs & .362 & .378 & & $2.485^{* *}$ \\
\hline Beliefs about norms & .314 & .303 & & $2.310^{*}$ \\
\hline \multirow[t]{2}{*}{ Goal beliefs } & .322 & .268 & & $2.194^{*}$ \\
\hline & \multicolumn{4}{|c|}{$\begin{array}{l}\text { Dependent variable: Rational (DSS) } \mathrm{R}=.322 \quad \mathrm{R}^{2}=.104 \\
\mathrm{~F}(\mathrm{df}=4 / 85)=2.46(\mathrm{p}=.052)\end{array}$} \\
\hline Beliefs about self & -.327 & -.305 & & $-2.080 *$ \\
\hline General beliefs & -.305 & -.317 & & $-1.751 *$ \\
\hline Beliefs about norms & -.237 & -.215 & & $-1.980 *$ \\
\hline Goal beliefs & -.211 & -.209 & & -1.560 \\
\hline
\end{tabular}

Note. $* \mathrm{p}<.05 \quad * * \mathrm{p}<.01 \quad * * * \mathrm{p}<.001$ 
In addition to providing a significant prediction of the four intuition variables the findings show that, as predicted, in each case all four $\mathrm{CO}$ beliefs had a significant contribution to the prediction. However, the relative contribution of each belief type differed in regard to the four intuition variables. Beliefs about self had the relatively highest contribution in regard to holistic and affective intuition, while norm beliefs had the highest contribution in regard to inferential intuition and general beliefs in regard to intuitive decision style.

Notably, in regard to rationality, which is the opposite of intuition, the CO beliefs accounted only for $10.4 \%$ of the variance. The contribution of all CO beliefs was negative. The contribution of goals was not significant.

The results concerning the factors based on motives confirm and expand the cited findings. The five factors provided a significant prediction of the four intuition variables, accounting on the average for $31.23 \%$ of the variance. All five factors had significant contributions to the prediction, which varied however across the variables. The factor of Emotions was related most highly to the affective intuition; the factors of Comprehensive view and of Opening-up were related most highly to holistic intuition; the factors of Self-reliance and Fast solutions were related most highly to inferential intuition. Again, the CO factors were related to rationality less than to the intuition variables and their contributions were negative.

\section{Discussion}

The results provided support to the hypothesis about the relation of $\mathrm{CO}$ variables to intuition by showing that the four CO beliefs enabled a significant prediction of four different intuition variables. Further, in regard to each prediction all four belief types had a significant contribution. These findings indicate that intuition is embedded in a network of personal tendencies and attitudes which constitute the motivational source for the disposition to rely on intuition in regard to the different domains of life. The earmarks of these motivationally relevant tendencies are focusing on emotions, opening-up to situations, preference for fast and easy solutions, promoting the overall comprehensive view of things and self-reliance. The power of these tendencies to orient toward intuition is due to the fact that they represent deep underlying meanings of intuition and operate in the form of a four-pronged vector defined by beliefs about self, general beliefs, beliefs about norms and beliefs about goals.

The structure of motivation allows however for some flexibility, both in regard to different forms of intuition as well as in regard to individual differences. The findings show that the relative weight of the four belief types as well as of the five motive factors changes in line with the different kinds of intuition. For example, as may be expected, the contribution of beliefs about self was largest in regard to the holistic and affective kinds of intuition. Similarly, it may be assumed that the relative contribution of the belief types or the motive factors to the prediction of the various intuition kinds may vary across individuals, in line with their personality traits and cognitive tendencies.

It is of particular interest to note that the belief types and factors provide a prediction also of rationality which may be considered as antithetical to intuition. As expected, the contribution of the three $\mathrm{CO}$ variables with a significant contribution is negative.

\section{Conclusions}

The study showed that intuition is cognitive tendency embedded in a motivational network of beliefs and meanings. As such it can be considered as a personality disposition. The findings provided a clear definition of the specific and unique components of the motivational groundwork of intuition, namely, reliance on oneself and especially on one's emotions, preference of a comprehensive overall view of issues and of fast solutions, complemented by opening-up to situations. This conclusion is not only theoretically important but has also practical implications. It enables assessing the motivational structure of intuition with a tool which does not suffer from social desirability because its items do not refer directly to intuition. Further, it enables devising a training program for promoting intuition when it is considered desirable. The training program will be based on strengthening the particular aspects of the $\mathrm{CO}$ of intuition identified in the study, in line with the systematic intervention procedure of the CO (Kreitler, 2004).

\section{References}

Gäbler, M. (1946). Diagnostic errors in diagnostic decision making. Wiener Medizinische Wochenschrift, 167, 333-342. [in German]

Hamilton, K., Shih, S.-I., \& Mohammed, S. (2016). The development and validation of the rational and intuitive decision styles scale. Journal of Personality Assessment, 98, 523-535. 
Hansson, P., Rönnlund, M., Juslin, P., \& Nilsson, L.G. (2008). Adult age differences in the realism of confidence judgments: overconfidence, format dependence, and cognitive predictors. Psychology and Aging, 23, 531-544.

Heintzelman, S. J., \& King, L. A. (2016). Meaning in life and intuition. Journal of Personality and Social Psychology, 110, 477-492.

Kahneman, D. (2003). A perspective on judgment and choice: mapping bounded rationality. The American Psychologist, 58, 697-720.

Kreitler, H., \& Kreitler, S. (1976). Cognitive orientation and behavior. New York: Springer.

Kreitler, H., \& Kreitler, S. (1982). The theory of cognitive orientation: Widening the scope of behavior prediction. In B. A. Maher, \& W. A. Maher (Eds.). Progress in experimental personality research (Vol. 11, pp.101-169). New York: Academic Press.

Kreitler, S. (2004). The cognitive guidance of behavior. In J. T. Jost, M.R. Banaji, \& D.A. Prentice (Eds.), Perspectivism in social psychology: The Yin and Yang of scientific progress (pp. 113-126). Washington, DC: American Psychological Association.

Kreitler, S. (2014). Predicting behavior: The cognitive orientation approach. In C. Pracana (Ed.), International psychological applications conference and trends (InPact) (pp. 99-102). World Institute for Advanced Research and Science (WIARS). Lisbon: Portugal.

Norris, P., \& Epstein, S. (2011). An experiential thinking style: its facets and relations with objective and subjective criterion measures. Journal of Personality, 79, 1043-1079.

Pretz, J. E., Brookings, J. B., Carlson, L. A., Humbert, T. K., Roy, M., Jones, M., \& Memmert, D. (2014). Development and validation of a new measure of intuition: The types of intuition scale. Journal of Behavioral Decision Making, 27, 454-467.

Rakoczy, H., Clüver, A., Saucke, L., Stoffregen, N., Gräbener, A., Migura, J., \& \& Call, J, (2014). Apes are intuitive statisticians. Cognition, 131, 60-68.

Razumnikova, O. M., \& Yashanina, A. A. (2017). Roles of rational-irrational cognitive styles in the alpha rhythm reactivity during convergent and divergent thinking. Rossiiskii Fiziologicheskii Zhurnal Imeni I.M. Sechenova, 103, 349-358 [in Russian]

Sagiv, L., Amit, A., Ein-Gar, D., \& Arieli, S. (2014). Not all great minds think alike: systematic and intuitive cognitive styles. Journal of Personality, 82, 402-417.

Thompson, V.A., Prowse Turner, J.A. \& Pennycook, G. (2011). Intuition, reason, and metacognition. Cognitive Psychology, 63, 107-140.

Appendix 1

The Cognitive Orientation Questionnaire of Intuitive Thinking

Note: In each of the following four parts of the questionnaire only 5 of the 18 items are presented for reasons of limited space. For the complete questionnaire, please write to Krit@netvision.net.il

Concerning each of the following statements please check how true it is: very true (4), true (3), not true (2) not at all true (1)

Beliefs about oneself: 1. In everything I do I focus mainly on the utilitarian-functional aspect; 2. I am curious only concerning very specific things; 3 . I like to open-up to things, to grasp the solution dictated by the situation; 4.I like fast and easy solutions; 5.I don't mind being sometimes wrong.

General beliefs: 1. Reason is able to control emotions; 2.it is impossible to rely on easy and fast solutions.; 3. Sensory experiences, such as colors, shapes, smells and tones can clarify the situation better than various theories; 4. It is better to let the situation to dictate to you the action than to impose on the situation a ready-made solution; 5 . The external impression is at least as important as the internal contents

Beliefs about norms: 1.It is necessary to deal with the comprehensive view rather than with the details; 2.One should let oneself flow with the situation, to experience the direction of the solution towards which the situation directs one; 3 . In order to delve deeply into the issue it is necessary to examine it thoroughly and systematically and not rely on intuition and generalized insights; 4.One should grasp thing as they are without trying to impose on them some structure or organization; 5. One should not flounder too much in regard to decisions

Beliefs about goals (to what degree do you want each of the following to be or continue): 1 . To be able to get simple and fast solutions; 2 . To strive to simple and clear things without complications; 3 . To be always able to focus on various examples and concrete demonstrations; 4 . To be able to open-up to a situation and let it dictate to me the desired solution; 5 . To let emotions rule me instead of reason 